\title{
Memory for unidentified items: Evidence for the use of letter information in familiarity processes
}

\author{
ANNE M. CLEARY and ROBERT L. GREENE \\ Case Western Reserve University, Cleveland, Ohio
}

\begin{abstract}
Participants can give accurate recognition judgments to word fragments that they are unable to complete. In three experiments, the generality of this finding was examined across tasks. Accurate memory judgments in the absence of identification were obtained in item recognition and judgments of presentation frequency but not in associative recognition or list discrimination. The former two tasks are thought to involve the use of familiarity; the latter two are thought to rely on recollection. The present results are consistent with the claim that recognition without identification reflects familiarity processes.
\end{abstract}

Previous research has shown that people can make reasonably accurate recognition judgments by using information only about the locations of specific letters within a given word (Peynircioğlu, 1990). Peynircioğlu presented participants with a list of words, followed by a test list containing four-letter word fragments. At test, people were asked not only to try to complete each word fragment, but also to rate the likelihood that each was from a word that had appeared in the study list. For fragments that people were unable to complete, ratings were significantly higher for those from studied words than for those from nonstudied words. That is, people were able to recognize that letter fragments had come from the study list, even when they could not identify their corresponding words.

It has long been thought that recognition may be based on two processes, one being familiarity and the other, the retrieval of study items (Mandler, 1980). Peynircioğlu's (1990) finding of recognition without identification would appear to be based most likely on familiarity. If people were using retrieval to make their recognition decisions in Peynircioğlu's study, it would seem that they would have been able to identify the words from which the fragments came. That is, if people were using a given fragment to retrieve aspects of the study episode that would be useful for making a recognition decision, they should also have been able to use the retrieved memory to complete the fragment. However, because people made reliable recognition decisions on fragments that they could not complete, recognition in Peynircioğlu's study seems to be best explained in terms of a familiarity process.

One way to determine whether the recognition without identification effect reflects the use of familiarity would

This research was conducted while A.M.C. was supported by the National Institute of Child Health and Human Development Mental Retardation Research Training Grant 5-T32-HDO7176-16. Correspondence concerning this article should be addressed to A. M. Cleary, Department of Psychology, Case Western Reserve University, 10900 Euclid Ave., Cleveland, OH 44106-7123(e-mail: amc16@ po.cwru.edu). be to determine whether it operates in a manner similar to that in which familiarity is thought to operate. In the experiments reported here, we addressed this question by examining the recognition without identification effect across various recognition tasks. Some recognition tasks are thought to involve the use of familiarity; others are thought primarily to involve retrieval. Therefore, our rationale was that if the effect reflects the use of familiarity, it should primarily occur in tasks typically thought to involve familiarity and should diminish in those thought primarily to involve retrieval.

Specifically, we attempted to find recognition without identification in item recognition, associative recognition, judgments of presentation frequency, and list discrimination. These tasks were chosen because they vary in the degree to which each is thought to tap into familiarity as opposed to retrieval. Whereas item recognition and frequency judgment tasks are thought to be sensitive to familiarity (see, e.g., Hintzman \& Curran, 1994; Hintzman, Curran, \& Oppy, 1992; Hintzman \& Hartry, 1990), associative recognition and list discrimination tasks are thought to be sensitive primarily to retrieval (e.g., Clark, Hori, \& Callan, 1993; Hintzman, Caulton, \& Levitin, 1998; Jacoby, 1991; Yonelinas, 1997). Therefore, if recognition without identification reflects the use of familiarity, it should primarily be found in item recognition and in frequency judgment tasks, and it should disappear, or at least diminish, in associative recognition and list discrimination.

\section{EXPERIMENT 1}

Associative recognition (the recognition that a pair of items has occurred together in a list) and item recognition (the recognition that a given item or items has occurred in a list) are thought to differ in terms of the relative contributions of familiarity and retrieval to performance. Although both processes undoubtedly play a role in both tasks, item recognition is often seen as being determined primarily by familiarity, whereas associative recognition 
is determined primarily by retrieval (Clark et al., 1993; Yonelinas, 1997). Much of the evidence on this point consists of functional dissociations that have been found between these two tasks. For example, in item recognition, an advantage for low-frequency words over highfrequency words is generally found, but this effect reverses in associative recognition (Clark, 1992; Clark \& Shiffrin, 1992). Item recognition and associative recognition exhibit different time courses during testing (Gronlund \& Ratcliff, 1989) and may be differentially affected by instructional manipulations during study (Hockley \& Cristi, 1996). If recognition without identification reflects the utilization of familiarity, it should be far more evident in item recognition than in associative recognition.

\section{Method}

Participants. Forty-eight Case Western Reserve University undergraduates participated individually to fulfill part of an introductory psychology course requirement.

Materials. The stimuli used in Experiment 1 were words and their corresponding unique two-letter fragments taken from Gibson and Watkins's (1988) set. So that they would resemble the stimuli that were used in Peynircioğlu's (1990) experiments, two additional letters were added to each fragment. If the first letter of a given fragment was not one of its two unique letters, one of the additional letters was the first letter of the word. Otherwise, the two additional letters were added at random. All stimuli were presented in capital letters.

Procedure. Each portion of the experiment was conducted on a computer. In both the item and the associative recognition conditions, each person participated in four study-test segments, and all study stimuli were presented at a 2 -sec rate.

Half of the participants were placed in the item-recognition condition. To maximize the likelihood that the recognition without identification effect would be replicated using the item recognition task, the procedure was designed to resemble Peynircioğlu's (1990) tasks as closely as possible. However, because it also served as a comparison with the associative recognition task, the study list for the item task consisted of word pairs rather than individual words. For this task, 120 words were paired with one another by a computer program, so that 60 pairs altogether were studied. The specific pairings of words were randomized across participants. However, this was after the words had been assigned to appear as the first or second members of a pair; the same set of words was designated to appear as the first, as opposed to the second member, for every participant. The program divided the study and test stimuli into a series of four study-test segments, so that 15 pairs of words were studied at a time, each followed by a test list of 30 word fragments. Fifteen of the test fragments corresponded to words presented in the study list ( 1 word out of each studied pair), and 15 belonged to new, nonstudied words. Stimuli were randomly assigned to studied and nonstudied conditions for each participant. For each question presented during testing, a word fragment first appeared at the top of the computer screen. The presentation of each word fragment was followed by two test questions. First, participants were asked to identify the word from which the fragment came. They could respond either by typing in a word at the cursor or by hitting the return key and leaving that question blank. After a response was given to the first question, the second question was presented. Here, participants were asked, "Did this word appear in the study list?" and were asked to give a rating using a scale of 0 to 10 ( 0 meaning definitely not studied and 10 meaning definitely studied). They were asked to respond by typing a number and hitting the return key. The participants were specifically instructed to rate every fragment, even if they were unable to identify the word from which it came.
For the associative version of the recognition task, to which the other half of the participants were assigned, the study list was presented in the same manner as in the item version. That is, 15 word pairs appeared in each of four study lists, and participants were given the same instructions. During testing, however, a total of 14 fragments were presented, each of which corresponded to a word from the study list. After a response was given to the identif ication question, participants were asked to indicate whether they thought that the fragment had been paired with a particular word in the study list. For example, they might be asked, "Did this word appear in the study list with the word amethyst? Again, participants were asked to give a rating using a scale of 0 to 10 ( 0 meaning definitely not paired with that word and 10 meaning definitely paired with that word) and were instructed to give ratings to all fragments, even those that they could not identify. For 7 of the 14 test fragments, the second question contained the word with which it had been paired in the study list. For the remaining 7, another study list word-that is, one with which it was not paired-was given in the second question.

In addition, because presenting each word's counterpart word from the study list might have served as a cue that would help people to identify the fragment, the participants were given a second chance to identify it after giving a rating. Specifically, they were asked, "Do you know what the word is now?" and could respond either by typing in a word, or by simply hitting the return key.

\section{Results and Discussion}

Although the primary focus of the present experiment was on recognition ratings for unidentified fragments, a few of its aspects replicated some well-documented phenomena in memory research. First, the results for the number of fragments people identified in the item task were consistent with prior studies that have shown priming with word fragment identification (e.g., Peynircioğlu, 1990; Tulving, Schacter, \& Stark, 1982). For the item task, people identified an average of $51 \%$ of the studied fragments and $27 \%$ of the fragments that were not from the study list $[t(23)=13.16, p<.001]$. For the associative task (in which all fragments came from studied words), people identified an average of $60.5 \%$ of the fragments.

Second, the results from the recognition ratings given in Experiment 1 are consistent with the finding that people attribute the ease with which a stimulus can be identified to its familiarity (e.g., Whittlesea, Jacoby, \& Girard, 1990). The means and standard deviations for the recognition ratings are presented in Table 1. This was shown for both the item and the associative recognition conditions; people's ratings tended to be higher for the fragments that were identified than for those that were not identified. For the item task, a $2 \times 2$ identification status (identified vs. unidentified) $\times$ old-new status (studied vs. nonstudied) repeated measures analysis of variance (ANOVA) showed a main effect of identification status on ratings $[F(1,22)=106.25, p<.001]$. This effect was also found for the associative task $[F(1,22)=51.81, p<.001]$.

In addition, the analysis for the item task showed a significant interaction between identification status and old-new status $[F(1,22)=41.77, p<.001]$, as did the analysis for the associative task $[F(1,22)=33.43, p<$ .001]. This interaction was such that the difference between ratings given to studied and nonstudied items (in the item task) and between intact pairs and repaired 
Table 1

Mean Ratings for All Experimental Conditions

\begin{tabular}{|c|c|c|c|c|c|c|c|c|}
\hline \multirow[b]{2}{*}{ Condition } & \multicolumn{4}{|c|}{ Identified } & \multicolumn{4}{|c|}{ Nonidentified } \\
\hline & $M$ & $S D$ & $M$ & $S D$ & $M$ & $S D$ & $M$ & $S D$ \\
\hline & \multicolumn{8}{|c|}{ Experiment 1} \\
\hline & \multicolumn{2}{|c|}{ Studied } & \multicolumn{2}{|c|}{ Nonstudied } & \multicolumn{2}{|c|}{ Studied } & \multicolumn{2}{|c|}{ Nonstudied } \\
\hline Item & 7.62 & 1.05 & 4.71 & 1.71 & 4.29 & 1.05 & 3.44 & 0.99 \\
\hline \multirow[t]{3}{*}{ Associative } & 7.82 & 1.47 & 4.02 & 1.98 & 3.71 & 1.07 & 3.51 & 1.02 \\
\hline & \multicolumn{8}{|c|}{ Experiment 2} \\
\hline & \multicolumn{2}{|c|}{ Repeated } & \multicolumn{2}{|c|}{ Nonrepeated } & \multicolumn{2}{|c|}{ Repeated } & \multicolumn{2}{|c|}{ Nonrepeated } \\
\hline \multirow[t]{3}{*}{ Frequency } & 7.24 & 1.07 & 5.13 & 1.27 & 3.97 & $\overline{1.14}$ & 3.57 & 1.21 \\
\hline & \multicolumn{8}{|c|}{ Experiment 3} \\
\hline & \multicolumn{2}{|c|}{ First Half } & \multicolumn{2}{|c|}{ Second Half } & \multicolumn{2}{|c|}{ First Half } & \multicolumn{2}{|c|}{ Second Half } \\
\hline List & 3.73 & $\overline{1.01}$ & 5.30 & $\overline{0.88}$ & 5.00 & $\overline{0.84}$ & 5.26 & 1.04 \\
\hline
\end{tabular}

items (in the associative task) was larger for the fragments that people were able to identify than for those that they could not. For ratings given to just the test items that people were able to identify, there was no significant interaction for old-new status (studied vs. nonstudied) $\times$ task (item vs. associative) $[F(1,45)=1.89$, n.s. $]$, nor was there a main effect of task on ratings $[F(1,45)=0.56$, n.s.].

Turning to the data of primary interest, a $2 \times 2$ task (item vs. associative) $\times$ old-new status (studied vs. nonstudied) mixed ANOVA design revealed a significant interaction for ratings given to unidentified fragments $[F(1,46)=7.57, p<.01]$. The difference between ratings given to studied and to nonstudied test stimuli was greater for the item recognition condition than for the associative recognition condition. In other words, the recognition without identification effect was greater in the item recognition condition. Because familiarity is thought to be a more useful basis for making item recognition than associative recognition decisions, this result supports the idea that recognition without identification reflects the use of familiarity in recognition. In further support of this notion, whereas a paired $t$ test showed a significant difference between ratings given to studied and to nonstudied unidentified fragments for the item task $[t(23)=6.82$, $p<.001]$, no such difference was found for the associative task $\left[t(23)=1.08\right.$, n.s. ${ }^{1}$

Because multiple study and test lists were used throughout the experiment, it is possible that people's ratings might have changed across lists. However, a $2 \times 4$ repeated measures ANOVA showed no significant interaction between ratings for old-new status (studied vs. nonstudied) and list (first, second, third, or fourth), for either the item task $[F(3,69)=0.57$, n.s. $]$ or the associative task $[F(3,69)=1.36$, n.s.]. Furthermore, there was no main effect of list on ratings given in either the item task $[F(3,69)=1.29$, n.s.] or the associative task $[F(3,69)=0.90$, n.s.]. Therefore, people's ratings do not appear to have changed across lists. Because multiple study lists were used in the subsequent experiments as well, these data were also analyzed for possible list effects and, as in Experiment 1, none were found. Therefore, this variable is not discussed further.

\section{EXPERIMENT 2}

Frequency judgment tasks are thought to be sensitive to familiarity (e.g., Hintzman \& Curran, 1995; Hintzman et al., 1992; Hintzman \& Hartry, 1990). According to some formal models (e.g., Hintzman, 1988), the familiarity signal evoked by a particular item will be proportional to the number of times it is repeated in the study list, leading to increased judgments of frequency with increased presentation frequencies.

Therefore, if the recognition without identification effect reflects the use of familiarity, it should be manifested in circumstances in which people are asked to give frequency estimates to items from the study list. We examined this in Experiment 2. Specifically, people were asked to rate the likelihood using a scale of 0 to $10(0$ meaning definitely only once and 10 meaning definitely three times) that a fragment came from a word that appeared once as opposed to three times in the study list. If recognition without identification is sensitive to presentation frequency, people's ratings should be higher for unidentified fragments of repeated words than for unidentified fragments of words that have not been repeated.

\section{Method}

Participants. Twelve Case Western Reserve University undergraduates participated individually in the experiment to partially fulfill an introductory course requirement.

Materials. The stimuli were the same as those used in Experiment 1 .

Procedure. The procedure was very similar to that used for the item task in Experiment 1, with a few exceptions. First, words were presented individually during study, rather than in pairs. Second, 30 words were presented at study, 15 of which were presented only once throughout the list and 15 of which were repeated three times; words were randomly assigned to these two presentation conditions for each participant. Third, all of the test fragments corresponded to words that had appeared at study; half of them came from words that were presented once and half came from words that appeared three times in the list. Fourth, when asked to give a rating to each fragment, people were instructed to rate the likelihood that the fragment came from a word that occurred once as opposed to three times throughout the study list. Therefore, they were asked the question, "Did this word appear once or three times?" They were to rate the fragment using a scale of 0 to 10 ( 0 meaning definitely once and 10 meaning definitely three times).

\section{Results and Discussion}

Again, as in Experiment 1, some well-documented phenomena were replicated in Experiment 2. First, people identified significantly more fragments of words that had been repeated three times $(61 \%)$ than of those that had been presented only once $(45 \%)[t(11)=8.64, p<.001]$. This result is consistent with prior studies that have shown that spaced repetitions in a study list lead to a greater priming effect than does a single presentation (e.g., Greene, 1990; Roediger \& Challis, 1992). Second, as in Experiment 1, recognition ratings tended to be higher for the fragments that were identified than for those that were not identified (see Table 1), as was evidenced by a $2 \times 2$ identification status (identified vs. unidentified) $\times$ 
repetition status (one time vs. three times) repeated measures ANOVA, which showed a main effect of identification status on ratings $[F(1,10)=122.92, p<.001]$. Also, as in Experiment 1, there was a significant interaction between identification status and old-new status, such that the difference between ratings given to once presented and three-times presented fragments was larger for those that people could identify than for those they could not identify $[F(1,10)=52.84, p<.001]$.

As for the data of primary interest-that is, ratings given to unidentified fragments-a paired samples $t$ test showed a significant difference between ratings given to fragments of words that were repeated three times and those given to fragments of only once presented words $[t(11)=2.24, p<.05]$. In other words, using a scale of 0 to 10 (zero meaning definitely presented once and 10 meaning definitely presented three times), people demonstrated memory for the frequency of items that they could not identify (see Table 1). Because familiarity is thought to be sensitive to presentation frequency, this result provided further support for the idea that recognition without identification reflects the use of familiarity.

\section{EXPERIMENT 3}

Both Experiments 1 and 2 supported the notion that recognition without identification is a manifestation of familiarity. In Experiment 3, we sought further support for this idea by examining its possible occurrence in yet another recognition task. Specifically, a list discrimination task was examined, whereby participants had to decide whether a particular test fragment had come from a word that was presented in the first or second half of the list. Accurate performance on this type of task is generally thought to require the use of recollection (Hintzman et al., 1998; Jacoby, 1991). Therefore, recognition without identification should not occur in list discrimination situations.

\section{Method}

Participants. Twenty Case Western Reserve University undergraduates participated individually in the experiment to partially fulfill an introductory psychology course requirement.

Materials. The same stimuli as in Experiments 1 and 2 were used in Experiment 3.

Procedure. The procedure used in Experiment 3 was the same as that for Experiment 2, with three exceptions. First, 15 words were presented in each of four study lists. Second, participants were tested with a total of 14 fragments for each test list, each of which came from the study list. Seven of these test fragments corresponded to words from the first half of the study list, and the other 7 came from words presented in the second half of the list. Words were randomly assigned to their positions in the study list for each participant. After being given the opportunity to identify a given test fragment, participants were asked to rate it on a scale of 0 to 10 (0 meaning definitely from the first half of the list and 10 meaning definitely from the second half of the list).

\section{Results and Discussion}

As with Experiments 1 and 2, a few aspects of the results from Experiment 3 demonstrate some well-documented findings in memory research. First, suggesting a primacy effect in the priming of word fragments, people identified significantly more fragments that came from the first half of the study list $(65 \%)$ than came from the second half of the study list $(57 \%)[t(19)=2.38, p<.05]$. Primacy effects have been shown to occur at least under some circumstances in word completion tests (Gershberg \& Shimamura, 1994).

Second, as in Experiments 1 and 2, people tended to give higher recognition ratings to fragments that they were able to identify than to those they could not identify (see Table 1), as was demonstrated by a $2 \times 2$ identification status (identified vs. unidentified) $\times$ list-placement status (first half vs. second half) repeated measures ANOVA, which showed a main effect of identification status on ratings $[F(1,19)=16.12, p<.01]$. The same analysis also showed a significant two-way interaction, such that the difference between ratings given to fragments from the first and second halves of the study list was larger for those that were identified than for those that were not identified $[F(1,19)=36.57, p<.001]$.

The data of primary interest, which were the ratings given to unidentified fragments, did not show a significant recognition without identification effect $[t(19)=1.25$, n.s.]. Given the usual assumption that familiarity has little role in making accurate list discrimination decisions (Hintzman et al., 1998; Jacoby, 1991), this result is consistent with the results of Experiments 1 and 2, in that recognition without identification appears to be a manifestation of familiarity.

Although there was no significant memory without identification, the mean ratings given to unidentified second-half items was higher than that given to unidentified first-half items. One should not rule out the possibility that a small amount of list discrimination is possible for unidentified items. Still, the experiment had sufficient power to detect an effect of the same magnitude as that found in item recognition. Experiment 3 had a .98 probability of finding a significant effect if the effect size was equal to that found in item recognition in Experiment 1.

\section{GENERAL DISCUSSION}

In the present study, we examined the possibility that Peynircioğlu's (1990) finding of recognition without identification reflects the use of a familiarity process. We attempted to find the effect in a number of recognition tasks thought to vary in the degree to which they rely on familiarity. Our logic was that if the effect reflects the use of familiarity, it should be detected only in tasks that are thought to tap into familiarity, and not in those thought to require recollection. Specifically, we searched for the effect in item recognition, associative recognition, a frequency judgment task, and a list discrimination task.

Under the assumption that the tasks that we used vary in the degree to which they each rely on familiarity, the results reported here support the notion that recognition without identification reflects familiarity. First, our results were consistent with past research that has suggested that 
familiarity plays a larger role in item recognition than in associative recognition (e.g., Clark et al., 1993; Yonelinas, 1997). The magnitude of the recognition without identification effect was greater in item recognition than in associative recognition. In fact, a statistically significant effect was not even detected in the associative version of the task. Second, in accord with many conceptions of familiarity (e.g., Hintzman, 1988; Hintzman et al., 1992; Hintzman \& Hartry, 1990), recognition without identification was shown to occur in the form of frequency estimates. Finally, supporting the assumption that list discrimination tasks require recollection (e.g., Hintzman et al.,1998; Jacoby, 1991), list discrimination was shown here to be relatively insensitive to the recognition without identification effect.

One may choose an alternative interpretation of these results-namely, that people are recollecting specific letter combinations and using this as a basis for making recognition judgments; however, such an account is inadequate for several reasons. First, people have no way of knowing, at the time of study, which letter combinations will be presented at test. This makes it difficult to see how people could follow a strategy of memorizing specific letter combinations. Second, the recognition without identification effect has been found when the study list is presented auditorily and when only two-letter fragments are presented at test (Cleary \& Greene, 2000; Peynircioğlu, 1990), making it even more difficult to see how such a strategy would be possible. Third, if people are able to recollect specific letter combinations and use them to make recognition judgments, they might also be able to recollect which words have been paired with which letter combinations and which letter combinations have appeared in which portions of a list. However, recognition without identification was not found in either of these two situations in the present study, as would have been expected if it resulted from the use of familiarity.

Because recognition without identification appears to be a manifestation of familiarity, it may provide clues about how the process works. The effect is broadly consistent with global matching approaches to the study of familiarity (Clark \& Gronlund, 1996; Gillund \& Shiffrin, 1984; Hintzman, 1988). That is, it fits into the traditional conception of familiarity as a signal that occurs after the features of a given test item have been matched with those of all of the studied items in memory. From this perspective, because people are recognizing on the basis of only a fraction of the features of an item, the effect seems to suggest that specific letters and their locations within a word may exemplify the kinds of features that can be used in the matching process of familiarity.

Second, recognition without identification appears to involve the use of features that are represented abstractly in memory. While it might be plausible that the letter location information used in recognition without identification is directly tied to the perception of the original study events, the effect does not appear to be data driven. If the effect were data driven, it would be expected to dis- appear in situations where the presentation modality has been changed from study to test (e.g., Roediger \& Blaxton, 1987; Roediger, Weldon, \& Challis, 1989). However, both Peynircioğlu (1990) and Cleary and Greene (2000) found the effect after presenting the study list in an auditory format and the test list in a visual format. Therefore, the representation of the letter location information used in recognition without identification appears to be abstract, suggesting that familiarity can operate on the basis of abstract feature representation.

\section{REFERENCES}

Clark, S. E. (1992). Word frequency effects in associative and item recognition. Memory \& Cognition, 20, 231-243.

Clark, S. E., \& Gronlund, S. D. (1996). Global matching models of recognition memory: How the models match the data. Psychonomic Bulletin \& Review, 3, 37-60.

Clark, S. E., Hori, A., \& Callan, D. E. (1993). Forced-choice associative recognition: Implications for global-memory models. Journal of Experimental Psychology: Learning, Memory, \& Cognition, 19, 871-881.

Clark, S. E., \& Shiffrin, R. M. (1992). Cuing effects and associative information in recognition memory. Memory \& Cognition, 20, 580598

Cleary, A. M., \& Greene, R. L. (2000). Recognition without identification. Journal of Experimental Psychology: Learning, Memory, \& Cognition, 26, 1063-1069.

Gershberg, F. B., \& Shimamura, A. P. (1994). Serial position effects in implicit and explicit tests of memory. Journal of Experimental Psychology: Learning, Memory, \& Cognition, 20, 1370-1378.

Gibson, J. M., \& WATKINS, M. J. (1988). A pool of 1,086 words with unique two-letter fragments. Behavior Research Methods, Instruments, \& Computers, 20, 390-397.

Gillund, G., \& Shiffrin, R. M. (1984). A retrieval model for both recognition and recall. Psychological Review, 91, 1-67.

Greene, R. L. (1990). Spacing effects on implicit memory tests. Journal of Experimental Psychology: Learning, Memory, \& Cognition, 16, 1004-1011.

Gronlund, S. D., \& RatclifF, R. (1989). Time course of item and associative information: Implications for global memory models. Journal of Experimental Psychology: Learning, Memory, \& Cognition, 15, 846-858.

Hintzman, D. L. (1988). Judgments of frequency and recognition memory in a multiple-trace memory model. Psychological Review, 95, 528-551.

Hintzman, D. L., Caulton, D. A., \& Levitin, D. J. (1998). Retrieval dynamics in recognition and list discrimination: Further evidence of separate processes of familiarity and recall. Memory \& Cognition, 26, $449-462$.

Hintzman, D. L., \& Curran, T. (1994). Retrieval dynamics of recognition and frequency judgments: Evidence for separate processes of familiarity and recall. Journal of Memory \& Language, 33, 1-18.

Hintzman, D. L., \& Curran, T. (1995). When encoding fails: Instructions, feedback, and registration without learning. Memory \& Cognition, 23, 213-226.

Hintzman, D. L., Curran, T., \& Oppy, B. (1992). Effects of similarity and repetition on memory: Registration without learning? Journal of Experimental Psychology: Learning, Memory, \& Cognition, 18, 667680.

Hintzman, D. L., \& Hartry, A. L. (1990). Commensurability in memory for frequency. Journal of Memory \& Language, 29, 501-523.

Hockley, W. E., \& CRISTI, C. (1996). Tests of the separate retrieval of item and associative information using a frequency-judgment task. Memory \& Cognition, 24, 796-811.

JACOBY, L. L. (1991). A process dissociation framework: Separating automatic from intentional uses of memory. Journal of Memory \& Language, 30, 513-541. 
MANDler, G. (1980). Recognizing: The judgment of previous occurrence. Psychological Review, 87, 252-271.

Peynircioğlu, Z. F. (1990). A feeling-of-recognition without identification. Journal of Memory \& Language, 29, 493-500.

Roediger, H. L., III, \& Blaxton, T. A. (1987). Effects of varying modality, surface features, and retention interval on priming in wordfragment completion. Memory \& Cognition, 15, 379-388.

Roediger, H. L., III, \& Challis, B. H. (1992). Effects of exact repetition and conceptual repetition on free recall and primed wordfragment completion. Journal of Experimental Psychology: Learning, Memory, \& Cognition, 18, 3-14.

Roediger, H. L., III, Weldon, M. S., \& Challis, B. H. (1989). Explaining dissociations between implicit and explicit measures of retention: A processing account. In H. L. Roediger III \& F. I. M. Craik (Eds.), Varieties of memory and consciousness (pp. 3-41). Hillsdale, NJ: Erlbaum.

Tulving, E., Schacter, D. L., \& Stark, H. (1982). Priming effects in word-fragment completion are independent of recognition memory. Journal of Experimental Psychology: Learning, Memory, \& Cognition, 8, 336-342.

Whitt lesea, B. W. A., Jacoby, L. L., \& Girard, K. (1990). Illusions of immediate memory: Evidence of an attributional basis for feelings of familiarity and perceptual quality. Journal of Memory \& Language, 29, 716-732.
YonelinAs, A. P. (1997). Recognition memory ROCs for item and associative information: The contribution of recollection and familiarity. Memory \& Cognition, 25, 747-763.

\section{NOTE}

1. Participants were given a second chance at identification in the associative recognition condition because this was the only condition in which they were given potentially useful information between the identification question and the recognition judgment. It should be noted that inclusion of this second chance at identification is not critical for our conclusions. An additional 12 participants were tested in associative recognition, using a procedure identical to that of Experiment 1 except that they were not asked to identify the stimulus again after associative recognition. On identified items, these participants gave a significantly higher mean rating to old items (7.77) than to new items $(4.96)[t(11)=$ $6.84, p<.001]$. However, for unidentified items, there was no difference in mean ratings of associative recognition between old items (3.76) and new items (3.72) $[t(11)=0.19$, n.s. $]$.

(Manuscript received March 21, 2000;

revision accepted for publication September 26, 2000.) 